\title{
Successful cord blood transplantation for mycosis fungoides.
}

Takuya Fukushima ${ }^{1}$, Kensuke Horio ${ }^{1}$, Emi Matsuo $^{1}$, Daisuke Imanishi ${ }^{1}$, Reishi Yamasaki $^{1}$, Hideki Tsushima ${ }^{1}$, Yoshitaka Imaizumi ${ }^{1}$, Koichi Ohshima $^{2}$, Tomoko Hata ${ }^{1}$, Shinichiro Yoshida ${ }^{3}$, Yasushi Miyazaki ${ }^{1}$, Masao Tomonaga ${ }^{1}$.

${ }^{1}$ Department of Hematology and Molecular Medicine Unit, Atomic Bomb Disease Institute, Nagasaki University Graduate School of Biomedical Sciences, Nagasaki, Japan, ${ }^{2}$ Department of Pathology, School of Medicine, Kurume University, Kurume, Japan, ${ }^{3}$ Department of Internal medicine, Nagasaki Medical Center, Ohmura, Japan.

Manuscript number: 07-220

Correspondence: Takuya Fukushima, M.D.

1-12-4 Sakamoto, Nagasaki 852-8523, JAPAN

Department of Hematology and Molecular Medicine Unit,

Atomic Bomb Disease Institute,

Nagasaki University Graduate School of Biomedical Sciences.

Tel.: +81-(0)95-819-7111, Fax: +81-(0)95-819-7113

E-mail: fukutaku@nagasaki-u.ac.jp

Running title: Cord blood transplantation for mycosis fungoides

Main category: Transplantation \& Cell Therapy

Subcategory: Allogeneic Transplantation

Type of manuscript: Letter

Type of computer: Win

Applications: Microsoft Word, Microsoft Powerpoint 
Mycosis fungoides (MF) is a cutaneous T-cell lymphoma associated with the invasion of transformed mature T-cells into the skin demonstrating polymorphic atrophic patches, plaques, to generalized erythrodermia. In general, the prognosis for advanced MF patients with metastasis to other sites has been reported to be poor even when treated with systemic therapies $[1,2]$. Several reports of allogeneic hematopoietic stem cell transplantation (allo-HSCT) with both myeloablative and reduced-intensity conditioning regimens [3-5] suggest the efficacy of allo-HSCT for MF through a graft-versus-lymphoma (GVL) effect [6].

In June 2004, a 26-year-old woman was admitted to our hospital because of generalized erythrodermia, a skin tumor of the head, and multiple lymphadenopathy. Her medical history started from 1996 with itchy erythema diagnosed as parapsoriasis in January 2001. Skin tumors developed 3 years later on the head, diagnosed as MF on biopsy. In June 2004, her disease status advanced with generalized multiple skin tumors and lymphadenopathy, eosinophilia (20\% of WBC), and the elevation of LDH (367 IU/L, normal range: 119-229). Lymph node biopsy and bone marrow analysis revealed the invasion of abnormal T cells, leading to a diagnosis of stage IV MF. Since 
systemic combination (biweekly CHOP, 8 cycles) or low-dose chemotherapy did not elicit any clinical response, allo-HSCT was considered appropriate for the treatment of this patient. In April 2005, allogeneic bone marrow transplantation from an unrelated donor was performed after reduced-intensity conditioning (fludarabine at $25 \mathrm{mg} / \mathrm{m}^{2} /$ day for 5 days and melphalan at $70 \mathrm{mg} / \mathrm{m}^{2} /$ day for 2 days) infusing $2.9 \times 10^{8}$ cells $/ \mathrm{kg}$ of bone marrow cells, which resulted in the rejection of donor cells. MF lesions that showed temporal regression after conditioning recurred within 5 weeks after transplantation. Another chemotherapy regimen with cladribine and etoposide did not lead to any improvement of MF after the first transplantation. She had multiple skin tumors with generalized erythrodermia and lymphadenopathy.

Considering the refractory nature of MF in this patient, we decided to perform a second allo-HSCT. In August 2005, after total body irradiation (12 Gy, 6 fractions) and cyclophosphamide (60 mg/kg/day, 2 days), cord blood $\left(2.2 \times 10^{7}\right.$ cells $/ \mathrm{kg}, \mathrm{HLA} 2$ loci mismatched, from a male donor) from the Japanese Cord Blood Bank Network was transplanted. For prophylaxis for graft-versus-host disease (GVHD), tacrolimus (0.03 $\mathrm{mg} / \mathrm{kg}$, continuous infusion) was used as a single agent. Neutrophils recovered on day 
14, and engraftment was confirmed in bone marrow by FISH analysis of sex chromosomes. Platelet recovery ( $>50,000 / \mathrm{mm}^{3}$ without transfusion) was observed on day 41. In terms of MF regions, skin tumors, erythrodermia, and lymphadenopathy began to diminish during conditioning, and disappeared by the time of engraftment, achieving clinical complete remission. Around day 85 after transplantation, skin tumors appeared again on both her legs with itchy skin regions, along with multiple duodenal ulcers (by endoscopic examination) and multiple areas of lymph node swelling (neck, axilla, mediastinum, and para-aorta by CT scan). Skin tumor biopsy confirmed the relapse of MF, and histological analysis of the duodenal ulcer strongly suggested EB virus-associated lymphoproliferative disease. Tacrolimus was reduced and discontinued within 2 weeks; then, skin tumors and skin lesions showed a gradual decrease in size and completely diminished by day 140 (Figure 1). No chemotherapy was added. There was no clear sign of acute or chronic GVHD even after the discontinuation of tacrolimus. There was no sign of MF on her skin and no lymphadenopathy on CT scan at more than 23 months after the second $\mathrm{CR}$, with a Karnofsky score of $90 \%$. 
Several groups described that neither conventional chemotherapy nor high-dose chemotherapy with autologous stem cell support was sufficient for the long-term remission of MF $[7,8]$. Based on the successful reports of allo-HSCT for MF and the efficacy of the withdrawal of immunosuppressants for some relapsed MF cases, the important role of the GVL effect for the control of MF is suggested $[9,10]$. This is the first report of successful CBT for advanced MF with the graft-versus-MF effect. Since cord blood is available for many patients through cord blood banks and the waiting period is relatively short, CBT could be a therapeutic option for MF patients who are candidates for allo-HSCT but lack suitable related or unrelated donors.

\section{References}

1. Diamondidou E, Cohen PR, Kurzrock R. Mycosis fungoides and Sezary syndrome.

Blood. 1996;88:2385-2409.

2. Kim YH, Hoppe RT. Mycosis fungoides and the Sezary syndrome. Semin Oncol. 
1999;26:276-289.

3. Burt RK, Guitart J, Taynor A, et al. Allogeneic hematopoietic transplantation for advanced mycosis fungoides: Evidence for a graft-versus-tumor effect. Bone Marrow Transplant. 2000;25:111-113.

4. Soligo D, Ibatici A, Berti E, et al. Treatment of advanced mycosis fungoides by allogeneic stem cell transplantation with a non-myeloablative regimen. Bone Marrow Transplant. 2003;31:663-666.

5. Molina A, Zain J, Arber DA, et al. Durable clinical , cytogenetic, and molecular remissions after allogenenic hematopoietic cell transplantation for refractory Sezary syndrome and mycosis fungoides. J Clin Oncol. 2005;23:6163-6171.

6. Jones RJ, Ambinder RF, Piatadosi S, et al. Evidence of a graft-versus-lymphoma effect associated with allogeneic bone marrow transplantation. Blood. 1991;77:649-653.

7. Bigler RD, Crilley P, Micaily B, et al. Autologous bone marrow transplantation for advanced mycosis fungoides. Bone Marrow Transplant. 1991;7:133-137. 
8. Olavarria E, Child F, Woolford A, et al. T-cell depletion and autologous stem cell transplantation in the management of tumor stage mycosis fungoides with peripheral blood involvement. Br J Haematol. 2001;114:624-631.

9. Rocha V, Wagner JE, Sobocinski KA, et al. Graft-versus-host disease in children who have received a cord-blood or bone marrow transplantation from an HLA-identical sibling. N Engl J Med. 2000;342:1846-1854.

10. Takahasi S, Ooi J, Tomonari A, et al. Comparative single-institute analysis of cord blood transplantation from unrelated donors with bone marrow or peripheral blood stem-cell transplants from related donors in adult patients with hematologic malignancies after myeloablative conditioning regimen. Blood. 2007;109:1322-1330. 
Figure legends

Figure 1.

Skin lesions of MF before CBT (A), and those after the discontinuation of tacrolimus (B).

Skin tumors (on the head, right eyelid, back, and upper arm) and erythroderma markedly improved (B). 
Figure 1.
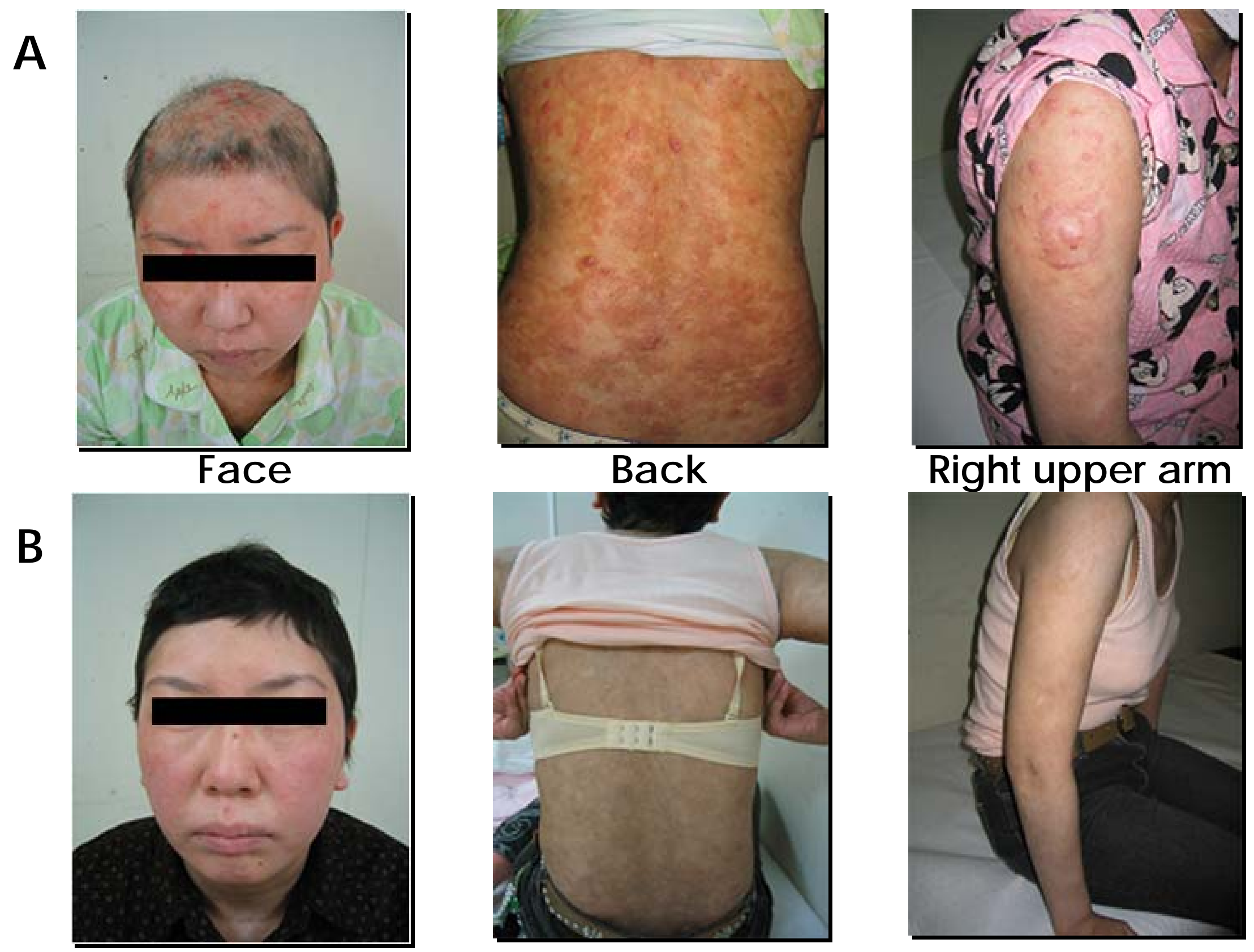